\section{Visualization of vitreomacular tractions with en face optical coherence tomography}

\begin{abstract}
Background In vitreomacular traction syndrome, an incomplete vitreous detachment with persistent vitreous traction on the macula is present. This condition may determine formation of epiretinal membranes, macular puckering, macular oedema, and traction macular detachment. Recently introduced en face optical coherence tomography (OCT) provides not only longitudinal B-scan but also coronal C-scan images of the retina. Methods Fifteen eyes of 11 non-diabetic patients presenting vitreomacular traction syndrome have been evaluated with en face OCT (OTI, Toronto, Ontario, Canada).
\end{abstract}

Results In 12 eyes, cystoid macular oedema was detectable at fundus examination and was associated with a broad-based adherence of the posterior hyaloid to the macula. In three eyes, foveal detachment appeared to be associated with focal foveal vitreoretinal traction. In all eyes, the lateral extent of the hyaloidal tractions on the macula was clearly detectable at coronal C-scan. They appeared as sigmoid hyper-reflective bands in the hyporeflective vitreous chamber. Overlay of C-scans on red-free confocal images allowed the visualization of the edges of the adherences in relation to the structures of the posterior pole. Conclusions The C-scan images allowed clear visualization of the total extension of the posterior vitreomacular adherences and their relationships with the vascular arcades, the optic disc, and the fovea.

Eye (2007) 21, 1391-1394; doi:10.1038/sj.eye.6702448; published online 2 June 2006

Keywords: vitreomacular traction syndrome; en face OCT; optical coherence tomography; OCT; vitreous detachment; foveal detachment
R Forte ${ }^{1}$, F Pascotto ${ }^{2}$ and $\mathrm{G}$ de Crecchio

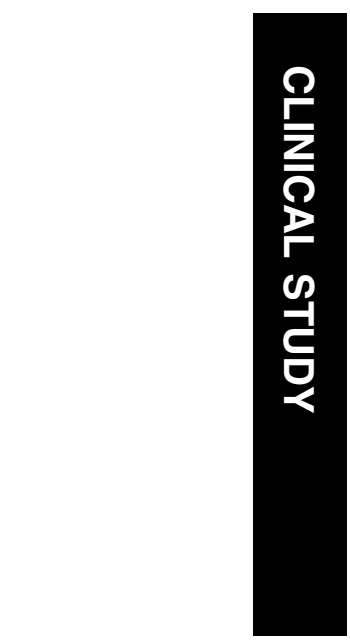

Introduction

In recent years, the important role of the vitreous in different macular diseases has been disclosed, ${ }^{1,2}$ and the introduction of optical coherence tomography (OCT) has allowed a fine visualization of the vitreoretinal interface. ${ }^{3}$ In vitreomacular traction syndrome, retinal changes develop from incomplete posterior vitreous detachment with persistent broad-based vitreous adhesion to the macula., ${ }^{4,5}$ This traction may determine formation of epiretinal membranes, macular puckering, and macular oedema with fluorescein leakage. If the base of the adherence is narrow, tractional cystoid not-leaking oedema ${ }^{6}$ and macular detachment may develop. The latter is considered as a first stage towards macular hole. ${ }^{7}$ In all these cases, vitrectomy and posterior hyaloid peeling have become an effective treatment in achieving resolution of retinal oedema and foveal detachment. ${ }^{8,9}$ En face OCT combines OCT and confocal ophthalmoscopy, allowing to obtain longitudinal B-scans and coronal C-scans of the retina. ${ }^{10-12}$ The confocal image, which has pixel to pixel correspondence with the coronal C-scan, allows a prompt visualization of the lateral extent of lesions on the posterior pole. Van Velthoven et $a l^{10}$ have extensively explained how to interpret coronal scans of the neuroretina obtained with this new technique in various macular diseases. In this study, we have evaluated vitreomacular traction syndrome with en face OCT.

\section{Methods}

We have considered 15 eyes of 11 non-diabetic patients presenting with visual reduction and vitreomacular traction. Best-corrected visual acuity, fundus examination, and en face
${ }^{1}$ Eye Department, University Federico II, Naples, Italy

2Eye Department, Second University, Naples, Italy

Correspondence: R Forte, Dipartimento di Scienze Oftalmologiche, Università Federico II, Via Pansini 5, 80131 Naples, Italy Tel: + 390817463731 ; Fax: + 390817462383 E-mail: raifor@hotmail.com

Received: 17 February 2006 Accepted in revised form: 23 April 2006

Published online:

2 June 2006 


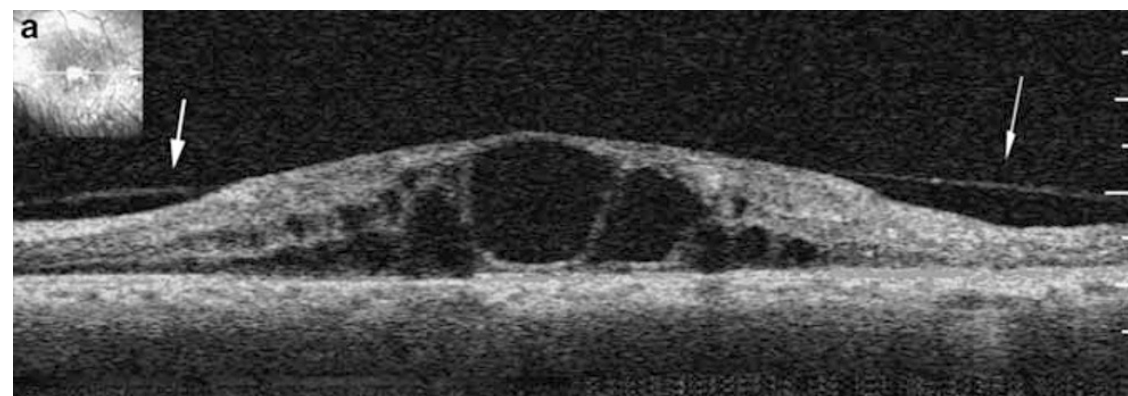

b

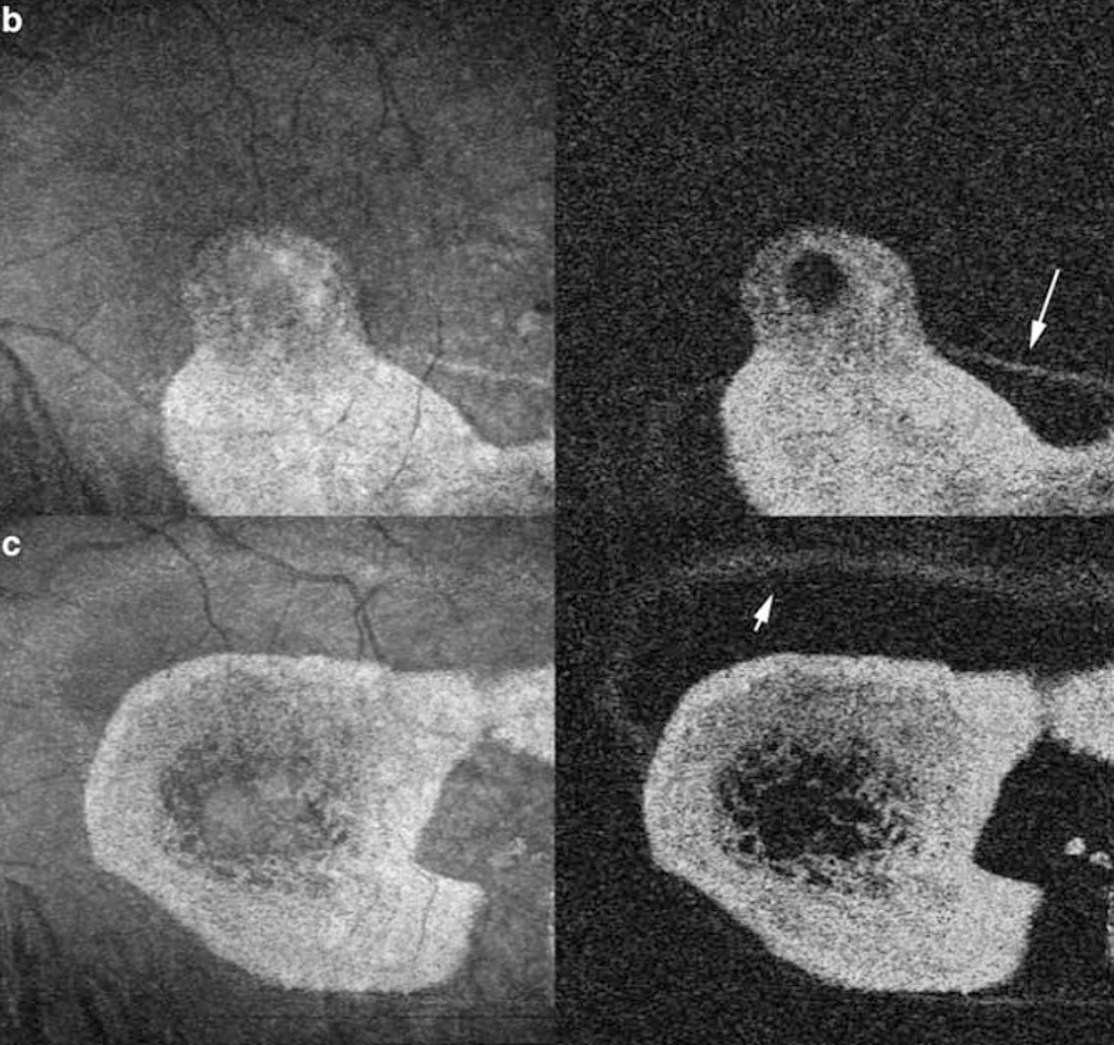

d

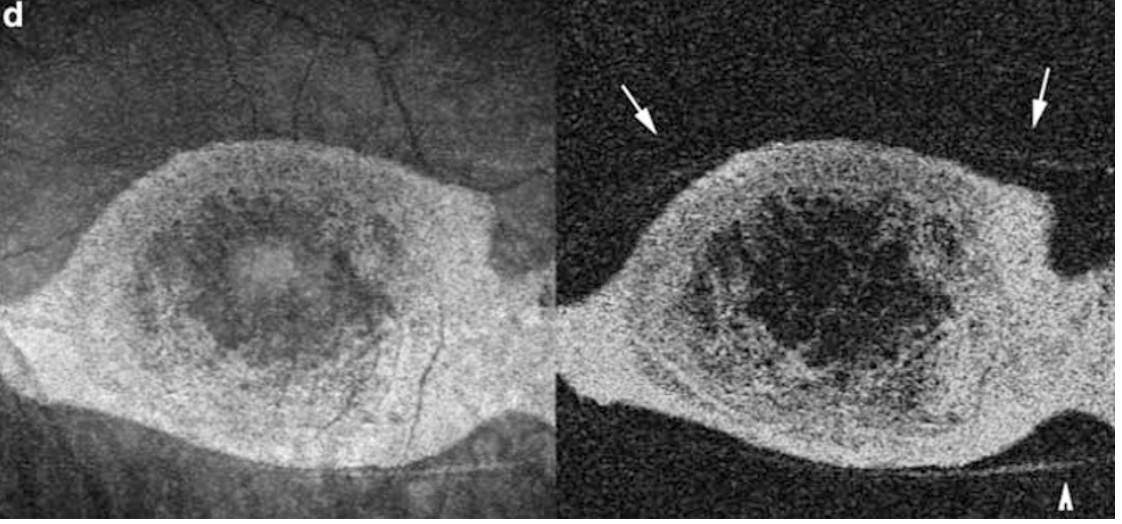

Figure 1 Cystoid macular oedema from broad-based vitreoretinal adherence at the posterior pole. (a) En face cross-sectional B-scan showing cystoid macular thickening with loss of foveal profile. The posterior hyaloid face can be seen as discrete linear bands (arrows) inserting into the fovea. (b-d) Left, overlaid red-free coronal C-scan image; right, OCT C-scan. Consecutive antero-posterior coronal OCT C-scans showing the macular cystoid changes and the sigmoid shape of the hyper-reflective adherent posterior hyaloid (arrows). The angular size is $20 \times 20^{\circ}$. 
OCT (OTI, Toronto, Ontario, Canada) have been performed in all cases.

\section{Results}

There were seven women and four men, with a median age of 46 (34-76) years. Visual acuity ranged from 0.1 $\log$ MAR (6/7.5 Snellen equivalent) to $1.0 \log$ MAR (6/60) (median acuity, 0.3 logMAR, 6/12), and metamorphopsia was present in all cases. In 12 eyes, cystoid macular oedema was detectable at fundus examination and was later confirmed at OCT (Figure 1). In all these cases, a broad-based insertion of the posterior hyaloid into the macula was detectable at cross-sectional B-scans as discrete hyper-reflective linear bands. In three eyes, ophthalmoscopic examination revealed a central yellow reflection and at OCT B-scan foveal detachment appeared to be associated with focal foveal vitreoretinal traction (Figure 2). In all examined cases, coronal OCT C-scans have shown the lateral extent of the hyaloidal bands at different depths from the vitreous to the vitreoretinal interface. They appeared as a low-to-moderate hyper-reflective sigmoid-shaped band in the hyporeflective vitreal chamber. At the level of the vitreous, because of the lack of point of reference, Adobe Photoshop ${ }^{\circledR}$ software has been used to reassemble multiple coronal slices in a global coronal view of the sigmoid bands (Figure 2e). At the level of the vitreoretinal interface, the overlay of coronal OCT scans on confocal red-free images has allowed to define the relationships among the vitreal tractions and the structures of the posterior pole.

\section{Discussion}

En face OCT has been recently introduced and different studies have shown its role in providing information not available with conventional OCT imaging. ${ }^{10,11}$ It allows not only longitudinal cross-sectional B-scans ( $10 \mu \mathrm{m}$ depth resolution) of the retinal layers, bearing strong resemblance to histology, but also coronal C-scans ( $15 \mu \mathrm{m}$ transversal resolution) of a retinal area at any given depth, enabling visualization of the lateral extent of structures. ${ }^{12}$ Visualization of epiretinal membranes with en face OCT shows a 'star-like' appearance. ${ }^{10}$ In this study, we used en face OCT to visualize macular oedema and foveal detachment from persistent vitreomacular traction. OCT coronal scans have allowed in all cases a fine visualization of the lateral extent and edges of the adherences of the posterior hyaloid, which appeared as a sigmoid hyper-reflective band in the black hyporeflective vitreous. Furthermore, the overlay of coronal C-scans on red-free confocal images has clearly shown the position of vitreoretinal adherences in relation to the structures of

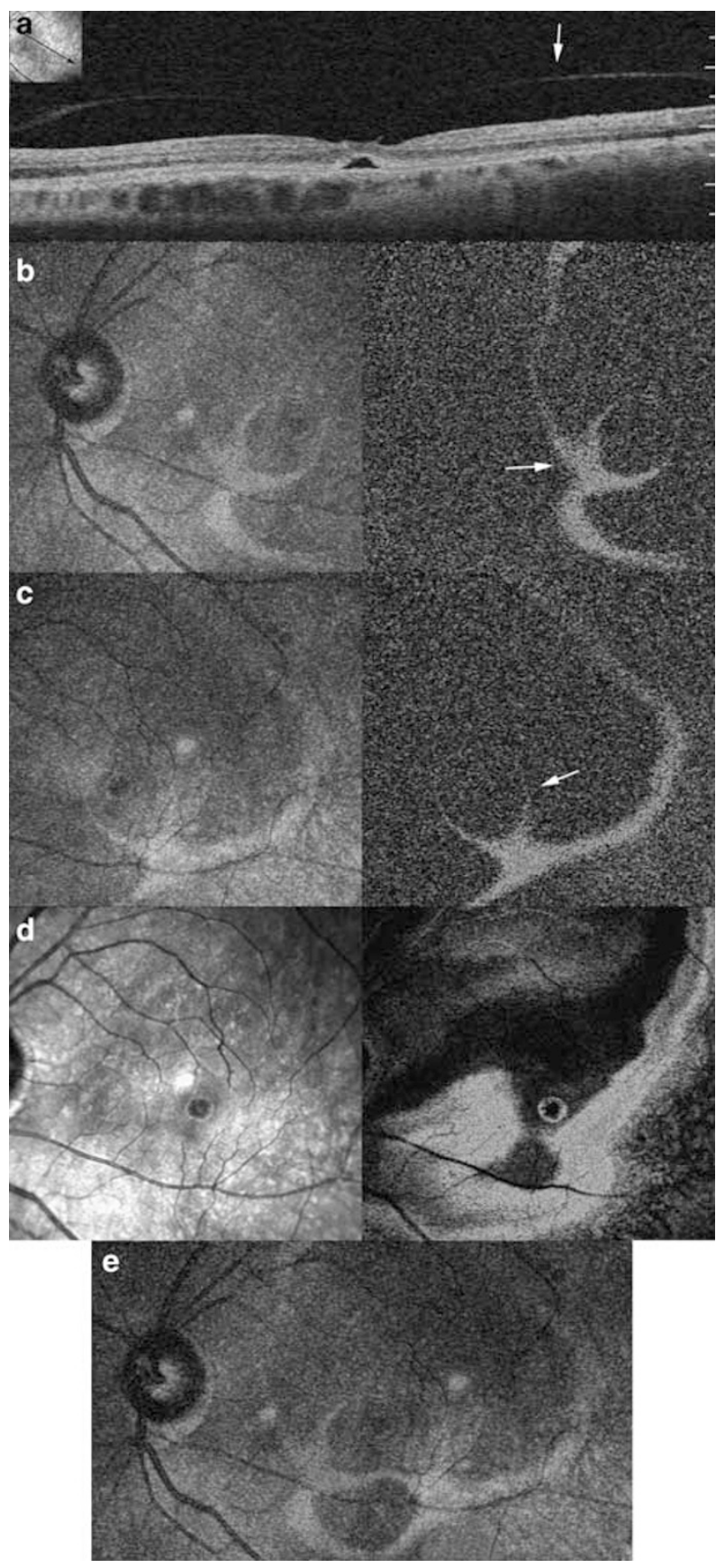

Figure 2 Foveal detachment from focal vitreoretinal adherence visualized with en face OCT. (a) En face longitudinal B-scan showing a dome-shaped non-reflective space under the fovea associated with loss of foveal contour and focal foveolar hyaloidal attachment (arrow). (b, c) Left, overlaid red-free coronal C-scan image; right, OCT C-scan. Consecutive anteroposterior C-scans. Angular size, $20 \times 20^{\circ}$. The sigmoid hyperreflective band and its relationships with the vascular arcades and the fovea are clearly detectable (arrow). (d) Left, red-free image; right, OCT C-scan. The hyporeflective circle represents the subretinal fluid. It is surrounded by the hyper-reflective ringshaped signal coming from the edges of the detachment. (e) Overlay of image $b$ and $c$ provides a global view of the vitreoretinal adherences. 
the posterior pole. As OCT does not compensate for the curvature of the eye, different ocular structures are imaged simultaneously at each coronal slice and interpretation of the whole amount of transverse scans has to be made. With regard to the retinal area, the mental reconstruction process is facilitated by the simultaneous confocal red-free image and by the presence of retinal vessels, which appear white if 'cut' by the OCT C-scans, or dark when coronal scans are taken more deeply into the retina, as the vessels cast a shadow on deeper layers. ${ }^{10}$ When scanning the vitreous and vitreoretinal interface, the lack of points of reference makes a software for the overlay of different slices useful for interpretation.

In conclusion, C-scan images can be used to obtain a three-dimensional reconstruction and a global view of the relationships between the retina and the vitreous. As surgery of focal attachments deserves sometimes difficulties in accessing to the subhyaloid space beca use of the fine dimensions of the localized perifoveal hyaloid detachment, ${ }^{6}$ these newly obtainable information about the vitreoretinal adherence can be important in planning the best approach to the posterior hyaloid for its removal.

\section{References}

1 Reese AB, Jones IS, Cooper WC. Vitreomacular traction syndrome confirmed histologically. Am J Ophthalmol 1970; 69(6): 975-977.
2 Sebag J. Anatomy and pathology of the vitreo-retinal interface. Eye 1992; 6(Part 6): 541-552.

3 Stanga PE, Bird AC. Optical coherence tomography (OCT): principles of operation, technology, indications in vitreoretinal imaging and interpretation of results. Int Ophthalmol 2001; 23(4-6): 191-197.

4 Jaffe NS. Vitreous traction at the posterior pole of the fundus due to alterations in the vitreous posterior. Trans Am Acad Ophthalmol Otolaryngol 1967; 71(4): 642-652.

5 Smiddy WE. Vitreomacular traction syndrome. In: Yanoff M, Duker JS (eds). Ophthalmology, 2nd ed. CV Mosby: St Louis, 2004, pp 951-955.

6 Johnson MW. Tractional cystoid macular edema: a subtle variant of the vitreomacular traction syndrome. $A m \mathrm{~J}$ Ophthalmol 2005; 140(2): 184-192.

7 Gass JD. Reappraisal of biomicroscopic classification of stages of development of a macular hole. Am J Ophthalmol 1995; 119(6): 752-759.

8 Subramanian ML, Truong SN, Rogers AH, Duker JS, Reichel E, Baumal CR. Vitrectomy for stage 1 macular holes identified by optical coherence tomography. Ophthalmic Surg Lasers Imag 2006; 37(1): 42-46.

9 Larsson J. Vitrectomy in vitreomacular traction syndrome evaluated by ocular coherence tomography (OCT) retinal mapping. Acta Ophthalmol Scand 2004; 82(6): 691-694.

10 Van Velthoven ME, Verbraak FD, Yannuzzi LA, Rosen RB, Podoleanu AG, DE Smet MD. Imaging the retina by en face optical coherence tomography. Retina 2006; 26(2): 129-136.

11 van Velthoven ME, Verbraak FD, Garcia PM, Schlingemann $\mathrm{RO}$, Rosen RB, de Smet MD. Evaluation of central serous retinopathy with en face optical coherence tomography. $\mathrm{Br}$ J Ophthalmol 2005; 89(11): 1483-1488.

12 Podoleanu AG, Dobre GM, Cucu RG, Rosen R, Garcia P, Nieto $\mathrm{J}$ et al. Combined multiplanar optical coherence tomography and confocal scanning ophthalmoscopy. J Biomed Opt 2004; 9(1): 86-93. 